\section{Aktinische Keratose: mit Photolyase neuen Läsionen besser vorbeugen}

\author{
Nach photodynamischer Therapie werden Patienten mit aktinischer \\ Keratose angehalten, das Hautareal mit Sonnencreme vor weiteren \\ UV-Schäden zu schützen. Dabei wäre eine Photolyase-haltige Sonnencreme \\ unter Umständen wirkungsvoller.
}

$P_{d}^{\text {h }}$ hotolyasen sind Enzyme, die UV-induzierte DNA-Schäden reparieren können. In der Langzeitanwendung haben sie sich bei Xeroderma-pigmentosaPatienten bewährt und das Neuauftreten aktinischer Keratosen (AK) und nicht melanozytärer Hauttumoren verringert. Warum sollten sie nicht auch bei AK-Patienten ohne Gendefekt zur Sekundärprävention wirksam sein?

Dermatologen aus Rom haben den Nutzen einer Photolyase-haltigen und photoprotektiv wirksamen Creme (Eryfotona AK-NMSC Fluid, Isdin, Spanien; kurz: Ery) getestet und mit dem einer herkömmlichen Sonnencreme (Avène Sonnencreme 50+, Laboratoires Dermatologiques Paris, Frankreich) verglichen. Teilgenommen haben insgesamt 30 immunkompetente Probanden zwischen 40 und 85 Jahren, die sich aufgrund einer aktinischen Keratose im Gesicht und/oder an der Kopfhaut einer photodynamischen Therapie (PDT) unterziehen wollten. Um nach einer PDT weiteren UV-Schäden vorzubeugen, sollte die eine Hälfte der Patienten neun Monate lang zweimal täglich (morgens und vier bis sechs Stunden später) die Sonnencreme auf das betroffenen Hautareal auftragen, die andere Hälfte das Ery-Fluid.

Zu Studienbeginn hatten die 30 Probanden insgesamt 225 AK-Läsionen. Das machte im Durchschnitt 7,5 Läsionen pro Proband. In der Ery-Gruppe waren es durchschnittlich 6,6 Läsionen und 8,4 Läsionen in der SonnencremeGruppe. Alle Probanden unterzogen sich einer PDT nach standardisiertem Protokoll mit Methyl-Aminolävulinsäure und einer 630-nm-LED-Lampe mit $37 \mathrm{~J} / \mathrm{cm}^{2}$. Zwei Wochen später wurden die AK-Läsionen erneut gezählt. Die Patienten der Ery-Gruppe brachten es auf durchschnittlich 2,0 Läsionen, die der Sonnencreme-Gruppe auf 0,6 Läsionen.

Nach neun Monate allerdings war in der Sonnencreme-Gruppe die Zahl der AK-Läsionen wieder deutlich gestiegen auf durchschnittlich 3,6, während in der
Ery-Gruppe die Zahl weiter rückläufig war und die Probanden im Durchschnitt nur noch 1,0 AK-Läsionen aufgewiesen haben. Während der Studie musste bei keinem Patienten der Ery-Gruppe erneut eine PDT oder eine andere großflächig angelegte Therapie durchgeführt werden, jedoch bei zehn Patienten, die sich mit Sonnencreme eingecremt hatten. Nebenwirkungen waren nicht aufgetreten.

Fazit: Für die Sekundärprävention ist eine Photolyase-haltige und photoprotektiv wirksame Creme deutlich effektiver als eine Sonnencreme allein. Die Ery-Therapie war mit einem deutlichen Rückgang neuer AK-Läsionen assoziiert, sodass anders als in der Sonnencreme-Gruppe eine weitere PDT oder eine andere flächenhafte Therapie nicht erforderlich waren. Angesichts des nicht doppelblinden Studiendesigns und der geringen Teilnehmerzahl wollen die Studienautoren die Ergebnisse jedoch mit Vorsicht interpretiert wissen.

Dr. Dagmar Kraus

Eibenschutz L et al. A 9-month, randomized, assessor-blinded, parallel-group study to evaluate clinical effects of film-forming medical devices containing photolyase and sun filters in the treatment of field cancerization compared with sunscreen in patients after successful photodynamic therapy for actinic keratosis. Br J Dermatol 2016; 175: 1391-3

\title{
Verdächtiges Ulkus an der Ferse
}

Ein 73-jähriger Mann stellte sich wegen einer Schrunde an der linken Ferse vor, die trotz regelmäßiger Pflege nicht abheilen wollte. Er wurde in eine dermatologische Ambulanz überwiesen, weil über dem linken Schienbein neu aufgetretene Knoten den Verdacht auf eine Mykose nahegelegt hatten. Hautbiopsien ergaben aber den Befund eines amelanotischen malignen Melanoms.

Die Manifestation des malignen Melanoms als Hautulkus ist ungewöhnlich. Die Inzidenz maligner Melanome beträgt 17,5/100.000. Eine Lokalisation an der unteren Extremität betrifft in $13 \%$ der Fälle Männer und in $39 \%$ Frauen. Nicht abheilende Ulzera und ungewöhnliche Hautknoten sollten frühzeitig biopsiert werden.

Prof. Hermann Füeßl

Shpadaruk V, Woo P. Non-healing leg ulcer. BMJ 2016; 352: i465

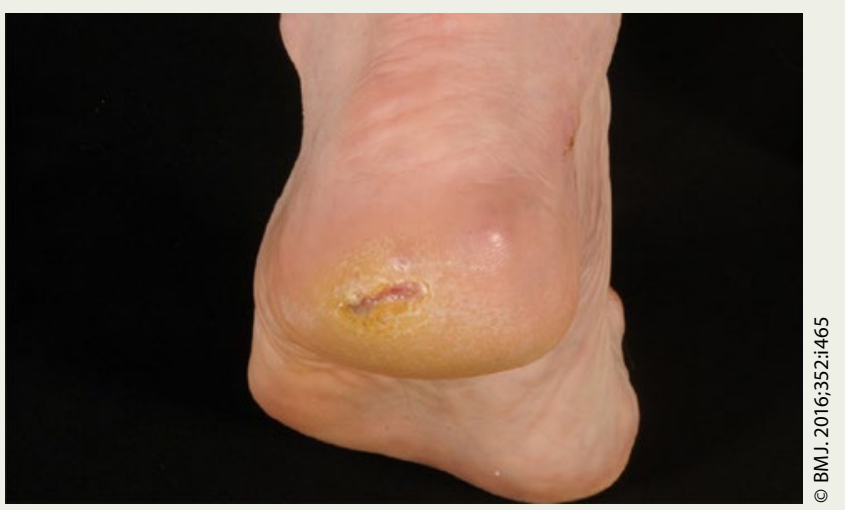

Nicht abheilende Schrunde an der Ferse 